\title{
Different Classifiers in Classification of Raw Arecanut
}

\author{
S Siddesha, S K Niranjan
}

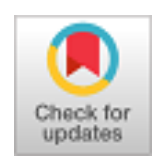

\begin{abstract}
Classification of crops is one of the important processes in precision agriculture. Classification of crops based on their verity, enhances the quality. In this paper, we presented a study of three main supervised classifiers, KNN, SVM and ANN for classifying the raw arecanut using color histogram and color moments as features. Experiments conducted over arecanut image dataset of 800 images across 4 classes. Among these classifiers K-NN gave a good result of $98.16 \%$ of with color histogram as feature.

Keywords: Color histogram, Color moments, Classification, KNN, ANN, SVM
\end{abstract}

\section{INTRODUCTION}

A recanut is one of the major cash crops of India. It has a major role in day to day life of every Indian family as a part of refreshment and also a part of tradition and culture. India is the number one country in the world in both production and consumption of arecanut. As per the study $47 \%$ of the word's production is from India and total productivity is 1.27 tones/ hectare [1]. It has a vital role in the preparation medicines in the field of ayurveda and veterinary. Nowadays we can see the usage of arecanut in areca tea. Classification of arecanut plays a role while grading. Proper classification helps is fixing the right price for right crop. From the survey it is evident that the works reported in this area were fragmentary. Few works reported are, automatic arecanut grading that focused on color, texture, and shape as features [2]. A work proposed having three steps, segmentation, masking, and classification for two class classification using red and green color has features after segmenting the area of arecanut [3]. A work reported on classification by studying texture features [4].

In first step for segmentation, global based threshold and Otsu methods are used followed by classification using mean and GLCM. A work was reported on grading using 3 sigma control limits on color features for segmentation with SVM for two grade boiling and Non-boiling classification [5].

Revised Manuscript Received on August 30, 2019.

* Correspondence Author

S Siddesha*, Department of Computer Applications, JSS Science and Technology University, Mysuru, India. Email: siddesh.shiv@gmail.com

S K Niranjan, Department of Computer Applications, JSS Science and Technology University, Mysuru, India. Email: siddesh.shiv@gmail.com

(C) The Authors. Published by Blue Eyes Intelligence Engineering and Sciences Publication (BEIESP). This is an open access article under the CC BY-NC-ND license (http://creativecommons.org/licenses/by-nc-nd/4.0/)

In our previous work, we classified the processed arecanuts using texture features with NN classifier [6]. The above reported classification work considered arecanuts without husk. This work mainly concentrates on classification of arecanuts into four classes.

\section{PROPOSED METHODS}

This model consists of three different stages. First stage is segmenting the crop from its background. This is to avoid shadow effect while capturing the image. These images segmented using K-means clustering method. The second stage used to extract features from the segmented image. Here color feature is extracted using color histogram and color moments techniques. In the third stage, the classification of arecanut into 4 classes is carried out. For classification we used three well known supervised classifiers K- Nearest Neighbor, Artificial Neural Networks and Support vector machine. A thorough experimentation is carried out by training the classifiers from $10 \%$ to $70 \%$ of training. After training, the test images are given in testing phase to match with the trained image. Upon proper matching the test image will be labeled with the class of the train image belongs to. This proposed model is shown in figure 1 .

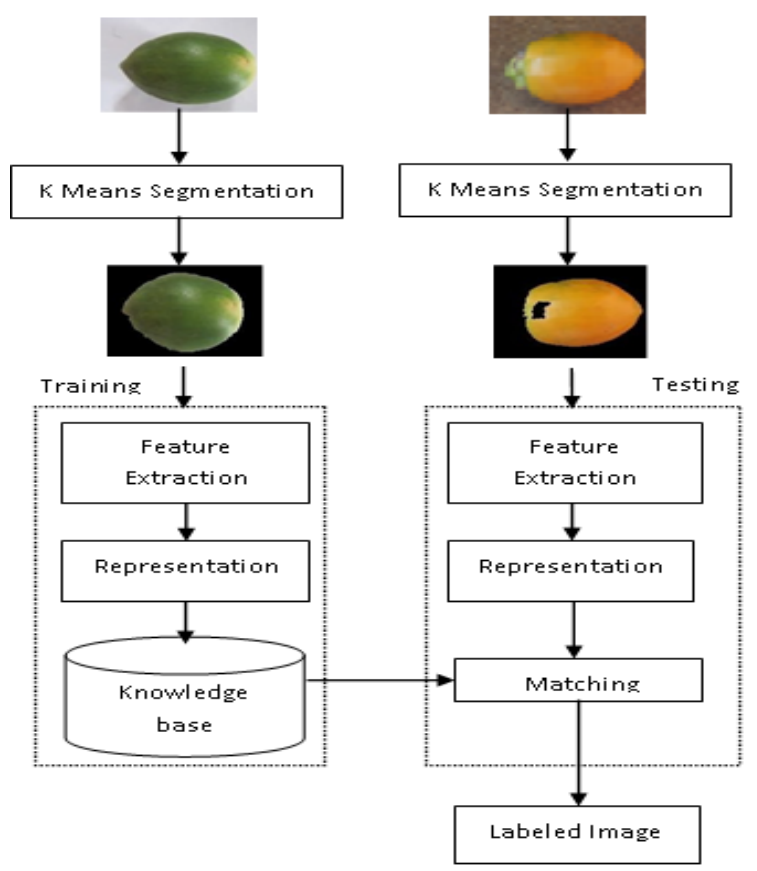

Fig. 1.Proposed model 


\section{Different Classifiers in Classification of Raw Arecanut}

\section{A. Segmentation}

Segmentation is the method of partitioning the image into sub parts so as to get the area of interest for further processing. The procedure of partition is carried out based on two premise properties of intensity, discontinuity and similarity [7].

- K-Means Clustering: Most commonly used basic unsupervised technique for low level image segmentation. This works on a specific number of disjoint partitions or clusters created which are nonhierarchical K-means doesn't require any intervention due to its unsupervised nature while separating the region of interest of an image from its background. It mainly concentrates on optimizing the process of joining partitions based on the cluster selected initially [8].

This algorithm divides the complex color textured images [9], [11]. It has a simple classification process of the given set of data with an apriori knowledge of $\mathrm{K}$ clusters. This algorithm updates space partition of the input data iteratively, by exchange of elements across clusters. This works on the basis of a predefined metric to satisfy the criteria to reduce the variation within each cluster and enhance the variation of $\mathrm{K}$ resulting clusters. This algorithm mainly follows four steps,

1. Initialization by defining number of clusters in the beginning and selecting the initial cluster centers randomly.

2. Creation of new partition by assigning each data point to the nearest cluster center.

3. Repeated calculation of center clusters for new data points and for clusters which are losing data points.

4. To meet a criterion for a distance convergence by repeating steps 2 and 3.

This method is a partitioning method which minimizes inter-group variance by combining objects. This yields an optimal dissimilarity of all global subsets by minimizing the dissimilarity in each local subset [7].

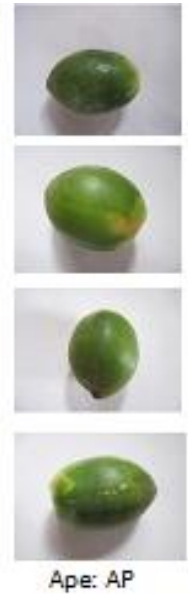

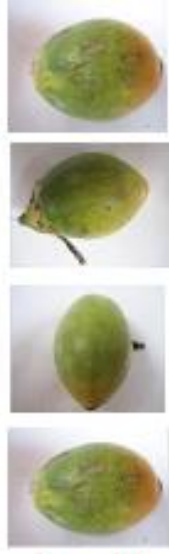

Bette: BT

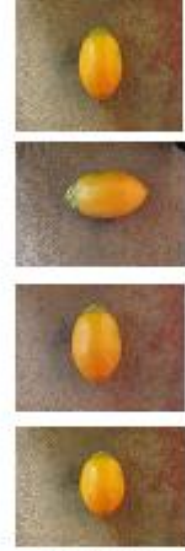

Mille: ML

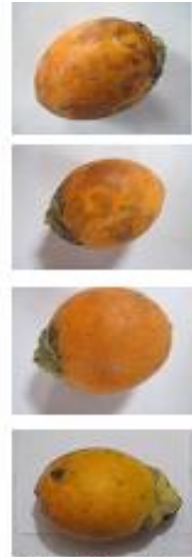

Gorabalu: GB
Fig. 2. Four classes of raw arecanut

\section{B. Feature Extraction}

Feature extraction is the process of extracting the information of an image for recognition, detection and classification tasks in computer vision and machine learning applications. Normally features are numerical values of the given image. Most common features are texture, color, shape, boundary etc [13]. Features are of global or local, whole image is described by global features and local features describe the key points of the image. Feature extraction is carried out after pre-processing step. This helps in discrimination of features from one class with other [12].

In this work RGB color model for arecanuts are considered as a global feature and extracted using color histogram and color moment techniques.

- Color histogram: Is most widely used feature extraction method for color image retrieval applications [14]. Normally this uses RGB color space. When an image is fed into this extraction method, color histogram is generated for each and every image from the given set of data. These histograms consist of proportion of each color pixels of the image [15]. For a color image histogram is the collection of bins, with each bin specifies the probability of specific color pixels of the image [16], and is as follows,

$$
C_{\text {Hist }}=C[0], C[1], C[3], \ldots \ldots . ., C[k], \ldots \ldots ., C[n]
$$

where $k$ is the color present in the histogram of an image and specifies the RGB Space sub cube. $C[k]$ is the number of pixels of a specific color of the given image $k$, and $n$ denotes the number of bins of generated color histogram. While generating the histogram for a given image, each pixel is mapped to a bin and each bin consists of number of pixels having the same color.

- Color histogram: Color moments are a metric to measure the similarity of color between the images. This is widely used in content based image retrieval applications. This works on the color probability distribution of an image, where the Probability distributions are characterized by a number of unique moments [17], [19], [20], [21]. The image can be identified by color by its probability distribution, which is nothing but different moments forms the features of the color. The features formed based on the probability distributions discriminates the color in one image with the other.

Once the similarity score computed between two images, the least score indicates that the images are similar. Here we used four color moments like mean, standard deviation, skewness and Kurtosis for the given arecanut image. There are 12 moments computed, 4 for each 3 color channels R, G and B. Let $C_{x y}$ defines the $x^{\text {th }}$ channel of color at $y^{\text {th }}$ image pixel. Then the four moments computed Mean (average color in the image), Variance (standard deviation computed through square root of the variance), Skewness (shape of the color distribution) and Kurtosis (the peakedness or flatness of the distribution) given by,

$$
\begin{array}{r}
C_{\text {mean }}=\sum_{y=1}^{N} \frac{1}{N}\left(C_{x y}\right) \\
\sigma_{x}=\sqrt{\left(\frac{1}{N} \sum_{y=1}^{N}\left(C_{x y}-C_{\text {mean }}\right)^{2}\right)} \\
\text { Skew }_{x}=\sqrt[3]{\left(\frac{1}{N} \sum_{y=1}^{N}\left(C_{x y}-C_{\text {mean }}\right)^{3}\right)}
\end{array}
$$




$$
\operatorname{Kurt}_{x}=\sqrt[4]{\left(\frac{1}{N} \sum_{y=1}^{N}\left(C_{x y}-C_{\text {mean }}\right)^{4}\right)}
$$

\section{Classification}

This is the final stage of the proposed model, which focuses on labeling the unknown arecanut image to the known finite classes based on similarity of features. Classification is a supervised learning technique, which has training and testing stage. In training stage, a sample is labeled and kept in a specific class. While testing, a unknown sample image will be compared with trained sample and if it is found similar to the training image, it is labeled with that of a training image.

In this work we used three different classifiers K-Nearest Neighbor (K-NN), Artificial Neural Network (ANN) and Support Vector Machines (SVM) and conducted experiments thoroughly.

- K-Nearest Neighbor (K-NN): K-nearest neighbor classifier is a non parametric supervised method. In K-NN classification, a sample is classified based on the majority votes of its neighbors, by assigning the label of the sample having most common of its neighbors [12]. The neighbors are considered from the set of samples with the knowledge of correct class. Different distance formulas are used to measure the similarities between the sample classes. Four distance measures Euclidean, Manhattan, Cosine and Chebychev are used measure the similarities and their effect on classification [18], [22], and these measures are given by,

$$
\begin{array}{r}
D_{\text {Еис }}(m, n)=\sqrt{\sum_{i=1}^{n}\left(m_{i}-n_{i}\right)} \\
D_{\text {Man }}(m, n)=\sum_{i=1}^{k}\left|m_{i}-n_{i}\right| \\
D_{\text {cos }}(m, n)=1-\frac{\sum_{i=1}^{k}\left(m_{i}-n_{i}\right)^{2}}{\sqrt{\sum_{i=1}^{k} m_{i}^{2}} \sqrt{\sum_{i=1}^{k} n_{i}^{2}}} \\
D_{\text {Che }}(m, n)=\max _{i}\left(m_{i}-n_{i}\right)
\end{array}
$$

- Artificial Neural Network (ANN): Neural networks are inspired by the operation of human brain. Neural networks are used in various types of pattern recognition applications. For classification purpose multilayer feed-forward network is used. These networks contain three layers input, hidden and output layers. Feed Forward Neural Network is a multilayer neural network having its signals moving unidirectional from input towards output layer. Before starting the network it needs to train on a set of data to compute the input-output mapping. This fixes the weights of short title 5 connections between units (neurons) then the network used for classifying the new data. At the time of classification, the signal of input units propagates through the net to compute the activation values in all output units. The activation values from each input unit will be sent to corresponding hidden units. These hidden units compute their own activation value and pass this signal to the output units. Each reviewing unit calculates the activation value as per the activation function. This function sums up the product of values of all units by defining the weight of connection from sending to receiving units with the activation value of sending units. Setting up the size of the hidden layer is problematic.

Less number of neurons may end up with poor approximation and excess may result in over fitting. The training of an ANN is normally carried out using back propagation method, which is used for updating of weights iteratively based on the error. The output layer has the error, which is the product of difference of expected with actual output and the slope of the sigmoidal activation function. The error is propagated back to the lower layers. The back propagation method is a descent algorithm trying to reduce the error in every iteration by adjusting the weights of the network. This is given by $\left(a_{l}, b_{l}\right), l=1,2,3, \ldots . ., L$ be the set of training pattern pairs. Here the main purpose is to find the weight update for each of an input-output pair. As the given data can be used many times during training, an index $m$ can be used to represent the training pair at step $m$. The training of a multilayer feed forward neural network can be given by the gradient descent to determine the weight increment connecting units $j$ and $i$ given by, [23],

$$
\Delta w_{i j}(m)=-\eta \frac{\delta E(m)}{i j}
$$

where $\eta>0$ and the weight update is given by,

$$
w_{i j}(m+1)=w_{i j}(m)+\Delta w_{i j}(m)
$$

In this work we used a three layer feed forward back propagation network and experimented by varying number of hidden layers from 1 to 3 .

Support Vector Machines (SVM): SVM is mainly devised for classifying a two class problem by separating the classes using creating hyperplane [24]. A hyperplane is a process of boundary fitting to a region of points which are similar. This makes the similar points to classify into a specific class. Once a hyperplane is created for training samples, for testing samples, we need to check whether they are within the same boundary or not. If it lies within the boundary it will be classified to a known created class. For a simple two attribute problem the hyperplane is either a straight line or a curve for separating them. The direct approach SVM for multi class problem is not we suited, so it will be avoided. For multi class problem different approaches will be employed [10]. A fair approach is by combining the several binary SVM classifiers, One among that is a One-Versus-One approach [25]. A multi category classification classifies the pattern by computing the $\mathrm{c}$ linear discriminant fucntions given by,

$$
g_{i}(x)=\omega^{\prime} x+\omega_{i 0}
$$

for $i=1,2, \ldots \ldots, c$ and assign $x$ to the category corresponding to the largest discriminant

A One-Versus-One classifier is constructed by dividing $\mathrm{n}$ classes into $n /(n-1) / 2$ binary classes. Each case will have a binary classifier responsible for dividing different pairs of classes. Learning is done by training the data with any of the two labels corresponding to the classes. If it encounters any class labels which are not similar, will be ignored. In the testing, a pattern is given to each one of the binary classifiers. 
The output is given by $S_{x y}$ in [01]. This provides confidence score of the binary classifier for dividing class $\mathrm{x}$ and $\mathrm{y}$ as per the previous class. The class having highest confidence scope will be the output class for the classifier [25]. The scope matrix of the classifier is given by,

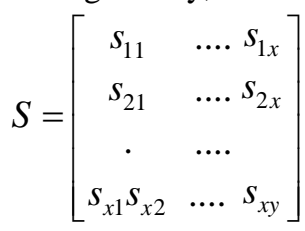

In SVM training is easy and operates nicely with dimensional data. Both continuous and categorical data can be easily handled. It predicts the nonlinear relations within the data and is a non-parametric technique. It deals well with erroneous data. The main problem with SVM is that it is complex to interpret features if they are uninterpretable. This made this computationally complex and to overcome a good kernel function is required [26], [27].

\section{DATASET AND EXPERIMENTATION}

Dataset is of very important for any computer vision and machine learning application for conducting experimentation. For arecanut crop there are no standard dataset available, we created our own dataset of 800 images divided into four classes with 200 images per class. Four classes considered in this work are 1: Ape (AP) 2: Bette (BT) 3:Minne (MN) and 4:Gorabalu (GB) shown in figure 2.These were captured at arecanut processing centers in and around Chitradurga district of Karnataka state in India. Nikon Coolpix L810 digital camera is used to capture these images by maintaining a distance of 1 meter. Dataset of 800 arecanut images across four classes are used for experimentation. The original images are segmented using K-Means clustering method to remove the shadow effect and then used for feature extraction. Two feature extraction methods color histogram and color moments are employed for extraction of color feature. For color moments four moments mean, Standard deviation, Skewness and Kurtosis were computed.

The extracted features in the form of feature vectors are stored in a folder. The classification is carried out using three different classifiers K-NN, ANN and SVM. While working with these classifiers we varied training from $10 \%$ to $70 \%$ and K values from 1 to 10 . In K-NN we explored the four different distance measures Euclidean, Manhattan, Cosine and Chebychev and studied their impact on classification accuracy. For color histogram features a maximum accuracy of 98.13\% achieved with Euclidean and Cosine distance formula and for color moment features a accuracy of 97.59\%with Chebychev distance for $\mathrm{K}=3$ for $20 \%$ training. The corresponding results are shown in figure 3 and 4.With ANN we used a feed forward network with back propagation mechanism for training. We trained the network by varying training samples from $10 \%$ to $70 \%$ and number of hidden layers from 1 to 3 . A maximum classification accuracy of $94.6 \%$ for color histogram features with 2 hidden layers and for $30 \%$ training and $93.8 \%$ of accuracy for color moment features with 3 hidden layers for $70 \%$ training is achieved and results are shown in figure 5 and 6, tables 1 and 2. In SVM we used three different kernel functions linear, polynomial and
RBF. While using One-Vs-One type of SVM a maximum accuracy of $94.38 \%$ for color histogram features for $40 \%$ training with linear kernel function and for color moment features $95 \%$ is achieved with polynomial kernel function with $40 \%$ training. The results are shown in table 3, 4 and figure 7,8 .

Among all the above classifiers, K-NN gave a good result. The reason behind this is the nature of dataset, which is not easily separable. The overlapping features of certain samples of one class with another makes it difficult to classify appropriately. In case of SVM generating hyperplane for multi classes is and in ANN training the network are the issues.

Table-I: Classification accuracy of ANN for Color Histogram features

\begin{tabular}{|c|c|c|c|}
\hline \multirow{2}{*}{$\begin{array}{c}\text { Training } \\
\mathbf{\%}\end{array}$} & \multicolumn{3}{|c|}{ Accuracy for different hidden layers } \\
\cline { 2 - 4 } & $\mathbf{1}$ & $\mathbf{2}$ & $\mathbf{3}$ \\
\hline 10 & 90.50 & 90.50 & 85.80 \\
\hline 20 & 91.70 & 92.20 & 91.70 \\
\hline 30 & 91.60 & $\mathbf{9 4 . 6 0}$ & 93.20 \\
\hline 40 & 92.10 & 93.50 & 91.90 \\
\hline 50 & 89.50 & 94.30 & 93.30 \\
\hline 60 & 91.60 & 95.30 & 92.20 \\
\hline 70 & 90.10 & 95.00 & 93.30 \\
\hline
\end{tabular}

Table-II: Classification accuracy of ANN for Color Moment features

\begin{tabular}{|c|c|c|c|}
\hline \multirow{2}{*}{$\begin{array}{c}\text { Training } \\
\mathbf{\%}\end{array}$} & \multicolumn{3}{|c|}{ Accuracy for different hidden layers } \\
\cline { 2 - 4 } & $\mathbf{1}$ & $\mathbf{2}$ & $\mathbf{3}$ \\
\hline 10 & 62.60 & 63.90 & 78.50 \\
\hline 20 & 69.40 & 87.70 & 85.20 \\
\hline 30 & 72.30 & 67.90 & 86.30 \\
\hline 40 & 70.60 & 68.50 & 88.30 \\
\hline 50 & 71.30 & 69.00 & 89.80 \\
\hline 60 & 68.10 & 71.90 & 90.60 \\
\hline 70 & 69.60 & 72.10 & $\mathbf{9 3 . 8 0}$ \\
\hline
\end{tabular}

Table-III: Classification accuracy of SVM with different Kernels for Color Histogram features

\begin{tabular}{|c|c|c|c|}
\hline \multirow{2}{*}{$\begin{array}{c}\text { Training } \\
\text { \% }\end{array}$} & \multicolumn{3}{|c|}{ Accuracy for different kernels } \\
\cline { 2 - 4 } & Linear & Polynomial & RBF \\
\hline 10 & 88.75 & 72.50 & 85.00 \\
\hline 20 & 70.63 & 71.32 & 89.38 \\
\hline 30 & 83.33 & 82.80 & 72.08 \\
\hline 40 & $\mathbf{9 4 . 3 8}$ & 92.50 & 93.44 \\
\hline 50 & 94.00 & 82.92 & 69.50 \\
\hline 60 & 93.13 & 82.17 & 89.17 \\
\hline 70 & 93.75 & 90.83 & 91.61 \\
\hline
\end{tabular}

Table IV: Classification accuracy of SVM with different Kernels for Color Moments features

\begin{tabular}{|c|c|c|c|}
\hline \multirow{2}{*}{$\begin{array}{c}\text { Training } \\
\text { \% }\end{array}$} & \multicolumn{3}{|c|}{ Accuracy for different kernels } \\
\cline { 2 - 4 } & Linear & Polynomial & RBF \\
\hline 10 & 60.17 & 73.75 & 60.23 \\
\hline 20 & 63.24 & 70.63 & 62.43 \\
\hline 30 & 62.81 & 81.25 & 62.08 \\
\hline 40 & 67.50 & $\mathbf{9 5 . 0 0}$ & 61.88 \\
\hline 50 & 80.31 & 80.25 & 72.75 \\
\hline 60 & 85.63 & 72.92 & 61.88 \\
\hline 70 & 77.14 & 90.36 & 78.21 \\
\hline
\end{tabular}


Table-V: Classification accuracy of KNN for K=3 with different distance formula for Color Histogram features.

\begin{tabular}{|c|c|c|c|c|}
\hline \multirow{2}{*}{$\begin{array}{c}\text { Training } \\
\text { \% }\end{array}$} & \multicolumn{4}{|c|}{ Accuracy for different distance formula } \\
\cline { 2 - 5 } & Eucl. & Man. & Cosine & Cheb. \\
\hline 10 & 94.03 & 93.06 & 85.69 & 92.22 \\
\hline 20 & $\mathbf{9 8 . 1 3}$ & 94.69 & 96.09 & 95.31 \\
\hline 30 & 98.04 & 98.04 & 98.04 & 95.71 \\
\hline 40 & 97.71 & 97.71 & 97.71 & 95.00 \\
\hline 50 & 97.75 & 97.75 & 97.75 & 94.50 \\
\hline 60 & 97.50 & 97.50 & 97.50 & 93.44 \\
\hline 70 & 96.67 & 96.67 & 96.67 & 91.25 \\
\hline
\end{tabular}

Table-VI: Classification accuracy of KNN with different distance formula for Color Moments features

\begin{tabular}{|r|c|c|c|c|}
\hline \multirow{2}{*}{$\begin{array}{r}\text { Training } \\
\mathbf{\%}\end{array}$} & \multicolumn{4}{|c|}{ Accuracy for different distance formula } \\
\cline { 2 - 5 } & Eucl. & Man. & Cosine & Cheb. \\
\hline 10 & 83.33 & 83.33 & 93.37 & 85.42 \\
\hline 20 & 96.41 & $\mathbf{9 7 . 5 6}$ & 89.69 & 97.59 \\
\hline 30 & 96.07 & 97.32 & 93.75 & 94.79 \\
\hline 40 & 95.42 & 96.75 & 92.71 & 96.25 \\
\hline 50 & 95.00 & 96.75 & 91.75 & 96.00 \\
\hline 60 & 94.06 & 96.25 & 90.00 & 95.31 \\
\hline 70 & 92.08 & 95.00 & 86.67 & 93.75 \\
\hline
\end{tabular}

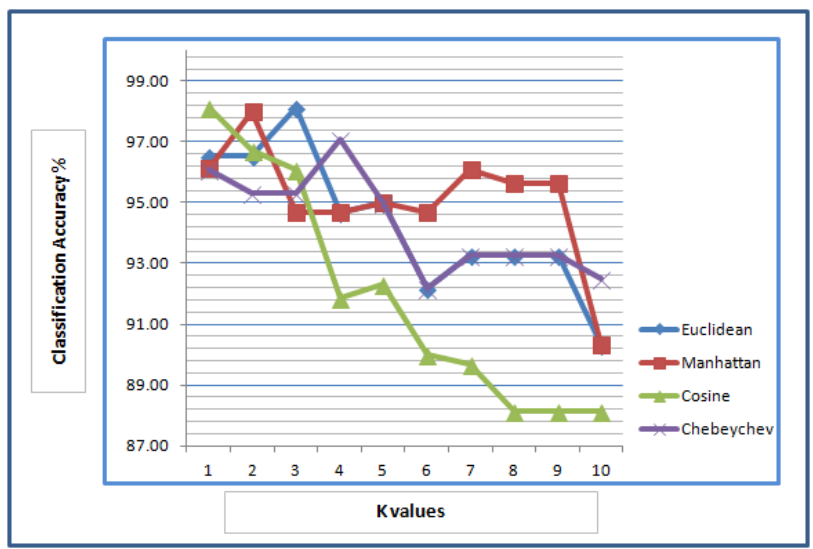

Fig. 3.KNN classification for colour histogram features

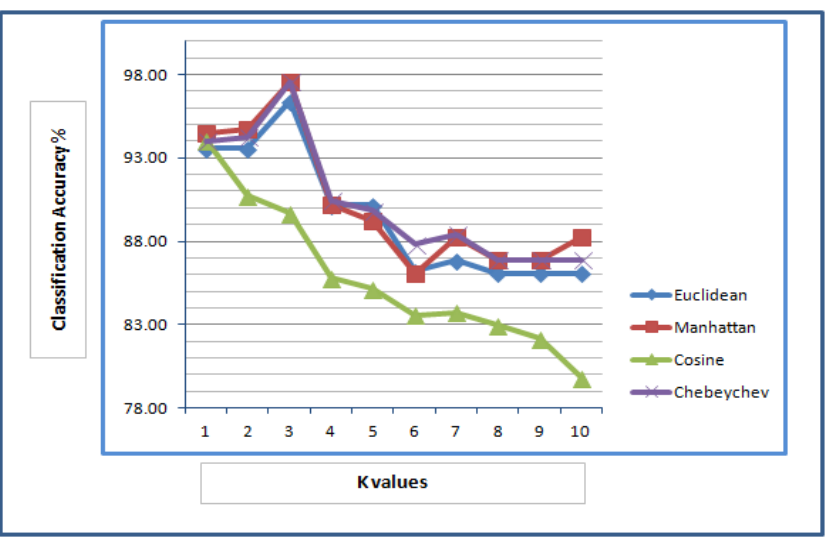

Fig. 4.KNN classification for colour moments features

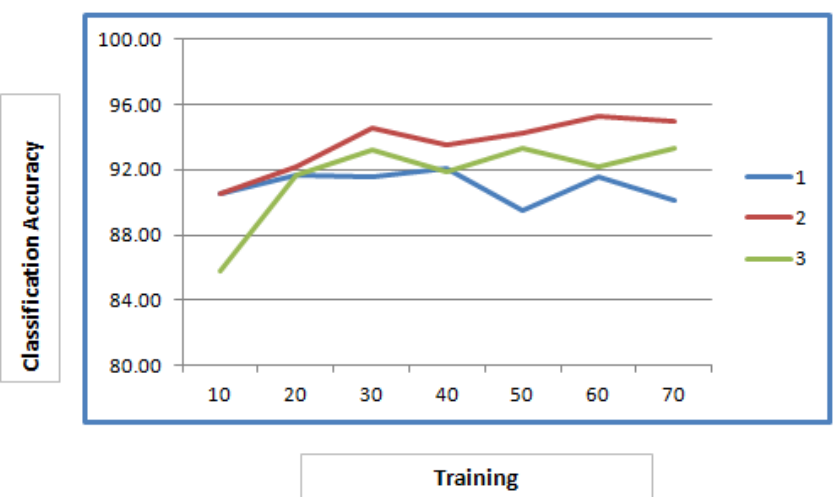

Fig. 5. ANN classification for color histogram features

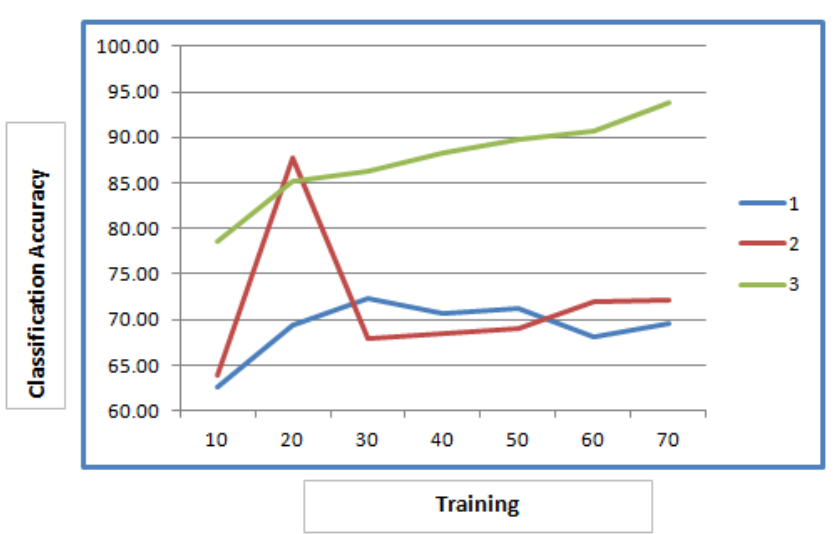

Fig. 6. ANN classification for color moments features

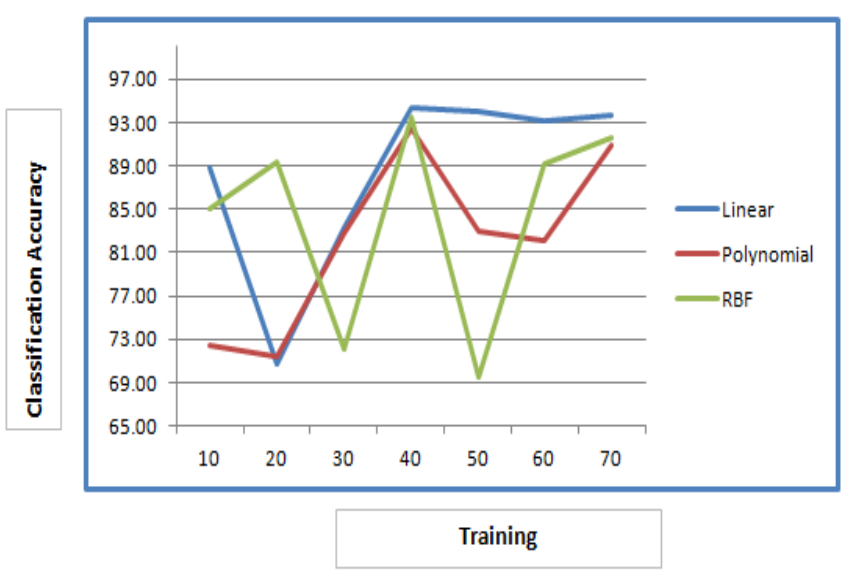

Fig. 7.SVM classification for color histogram features

\section{CONCLUSION}

This work deals with classification of raw arecanut based on color features with three classifiers K-NN, ANN and SVM.

Experimentation conducted on KNN classifier with four distance measures to analyze the impact. Classification of raw arecanut using color histogram gave good result of $98.13 \%$ with KNN having $\mathrm{K}=3$ for $20 \%$ training and for Euclidean distance measure. From figure 3 and 4, we can see little difference in classification accuracy.

Published By:

Blue Eyes Intelligence Engineering \& Sciences Publication

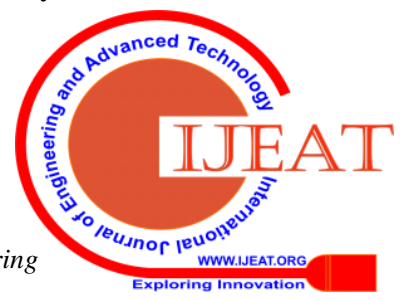


With ANN classifier the experimentation results shows a good classification accuracy of 94,6\% for histogram features with $30 \%$ training when used 2 hidden layers and $93.8 \%$ using color moment features with $70 \%$ training for 3 hidden layers. Results are shown in figure 5 and 6, tables 1 and 2. In case of One-versus-One SVM with linear kernel function maximum classification accuracy of $92.5 \%$ for histogram and $85.63 \%$ for color moments features achieved. Results are shown in table 3, 4 and figure 7, 8. Further we can extract other features along with color features and use of deep learning techniques for improvement.

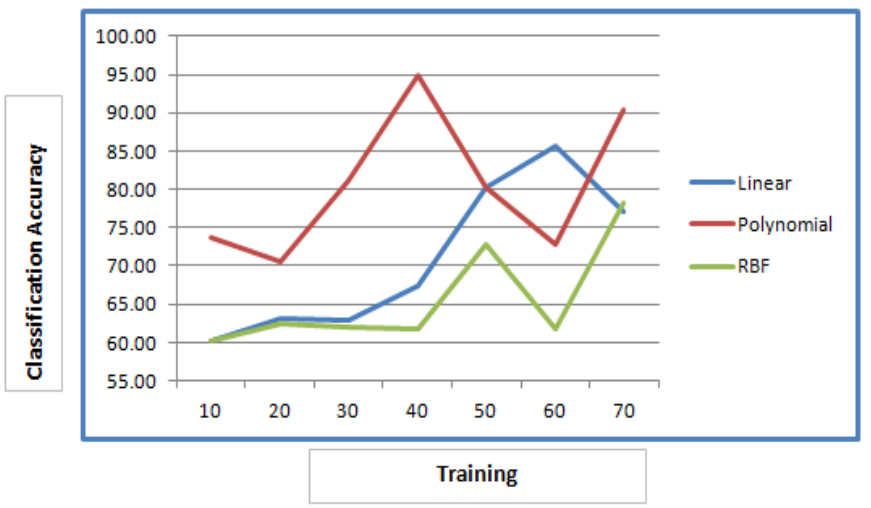

Fig. 8.SVM classification for color moments features

\section{REFERENCES}

1. C. P. Gracy, N. Nagashree, A. Nayak, and K. Girisha, K. (2010). Recent Posts on Indian Scenario of arecanut, http://krishisewa.com $/ \mathrm{cms}$ /articles/productiontechnology/61-arecanut.html/.

2. K. Liu, J. Xie, Y. He, M. Xu, and C. Qin. An Automatic Classification Method for Betel Nut Based on Computer Vision, Proceedings of the IEEE International Conference on Robotics and Biometrics, Guilin, China, 2009, pp.19-23.

3. A. Danti and M. Suresha. Segmentation and classification of raw arecanut based on three sigma control limits'. Proceedings of the IEEE International Conference on Robotics and Biometrics, Journal Procedia Technology, Sciverse Science Direct, Vol. 4, 2012,pp.215-219.

4. A. Danti and M. Suresha. Texture Based Decision Tree Classification for Arecanut', Proceedings of the CUBE International Information Technology Conference, ACM Publications, 2012, pp.113-117.

5. A. Danti and M. Suresha. Arecanut Grading Based on Three Sigma Controls and SVM, IEEE-International Conference On Advances In Engineering, Science And Management (ICAESM), Nagapattinam, Tamilnadu, India, 2012,pp.372-376.

6. S. Siddesha, S.K. Niranjan, V.N.M Aradhya. Texture based classification of Arecanut, Proceedings of International Conference on Applied and Theoretical Computing and Communication Technology (iCATccT), 2015, pp.1-18.

7. R.C. Gonzalez and R.E. Woods. Digital Image Processing, 3rd Edition, Pearson Education, 2009, pp.523-525.

8. T. Kanungo, D.M. Mount, N.S. Netanyahu. An efficient k-means clustering algorithm: analysis and implementation, IEEE Transactions on Pattern Analysis and Machine Intelligence, vol.24, Issue.7, 2002, pp.881-892.

9. M. Celenk. A color clustering technique for image segmentation, Journal of Computer Vision, Graphics, and Image Processing, 52, 1990, pp.27-34.

10. K. Crammer, Y. Singer. On the Algorithmic Implementation of Multiclass Kernel based Vector Machines, Journal of Machine Learning Research, 2, 2001, pp.265-292.

11. Y. Xi, D.D. Feng, T. Wang, R. Zhao and Y. Zhang. Image segmentation by clustering of spatial patterns, Pattern Recognition Letters, 28, 2007, pp.1548-1555.

12. R.O. Duda, P.E. Hart, D.G. Stork. Pattern Classification, Second Edition, Wiley India, 2012, pp.174-184.

13. D.P. Tian. A Review on Image Feature Extraction and Representation Techniques, International Journal of Multimedia and Ubiquitous Engineering, Vol. 8, No. 4, 2013, pp.385-396.
14. M. Stricker, M. Swain. The Capacity and the Sensitivity of Color Histogram Indexing, Technical Report, 94-05, 1994, pp.1-18.

15. S.R. Kodituwakku, S. Selvarajah. Comparison of Color Features for Image Retrieval, Indian Journal of Computer Science and Engineering,Vol. 1 No. 3, 2004, pp.207-211.

16. M.J. Swain, D.H. Bellard. Color Indexing, International Journal of Computer Vision, 7:1, 1991,pp.11-32.

17. M. Stricker, M. Orengo. Similarity of color images, Proceedings Volume 2420, Storage and Retrieval for Image and Video databases III, 1995,pp.1-12.

18. L.Y. Hu, M.W. Huang, S.W Ke, C.F. Tsai. The distance function effect on k-nearest neighbor classification for medical datasets, J. Springer Plus,5(1)-1304, 2016, pp.1-9.

19. S. Ghosal, R. Mehrotra. A Moment-Based Unified Approach to Image Feature Detection. IEEE Transactions on Image Processing, Vol. 6, No. 6, 1997, pp.781-793.

20. M.K. Mandal, T. Aboulnasr, S. Panchanathan. Image indexing using moments and wavelets, IEEE Transactions on Consumer Electronics, Vol.42, Issue: 3, 1996, pp.557-565.

21. A.Vailaya, M. Figueiredo, A. Jain. Content-based hierarchical classification of vacation images, Proceedings IEEE International Conference on Multimedia Computing and Systems, 2002, pp.1-15.

22. V.B.S. Prasath, H.A.A. Alfeilatb, O. Lasassmehb, A.B.A. Hassanat. Distance and Similarity Measures Effect on the Performance of K-Nearest Neighbor Classifier, A Review, Journal CoRR abs 1708.04321, 2017, pp.1-50.

23. Yegnanarayana, B. Artificial Neural Networks, Seventh Printing, PHI India, 2010, pp. 15-39.

24. B. Boser, I. Guyon, V. Vapnik. A training algorithm for optimal margin classifiers. In: Fifth Annual Workshop on Computational Learning Theory, Pittsburgh, ACM, 1992, pp.144-152.

25. Y. Ahuja, S.K. Yadav. Multiclass Classification and Support Vector Machine, Global Journal of Computer Science and Technology Interdisciplinary, Volume 12, Issue 11, Version 1.0, 2012, pp.15-19.

26. V. Jakkula, Tutorial on support vector machine (svm). School of EECS, Washington State University. 2006, pp.1-18.

27. L. Auria, and R.A. Moro. Support vector machines (svm) as a technique for solvency analysis, Discussion paper. 2008, pp.1-18.

\section{AUTHORS PROFILE}

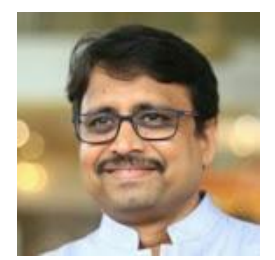

S. Siddesha is working as Assistant Professor in the Department of Computer Applications at JSS Science and Technology, Mysuru, Karnataka, India. He received his Bachelor of Science (B.Sc), Master of Computer Applications (MCA) and Master of Science and Technology (by Research) from University of Mysore. Currently he is perusing Ph.D in Visvesvaraya Technological University, Belagavi, Karnataka, India. His research interests include Computer vision and Machine learning.

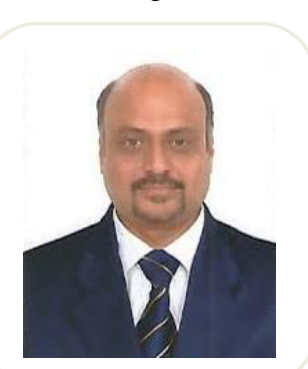

Dr. S $\mathbf{K}$ Niranjan is working as professor in the Department of Computer Applications at JSS Science and Technology, Mysuru, Karnataka, India. He has more than 25 years of experience both in teaching and industry. He received his Bachelor of Business Management (BBM), Master of Computer Applications (MCA), Master of Technology in Software Engineering (M.Tech [SE]) and Ph.D in Computer Science from University of Mysore. His interested are Image Processing, Pattern Recognition, Software Engineering, Business Analytics and Intelligence etc. More than 50 technical papers have been published by him in both national and international books, journals and conference. He was a General Chair for around six intentional conferences conducted in different parts of India. He is an active member of organizations like IEEE, ACM, CSI, ISTE etc. He Served as Executive Committee member in 2015 and 2016 for IEEE Computer Society, India Council. Currently he is an active member of IEEE Bangalore Section and Executive Committee (EXECOM).

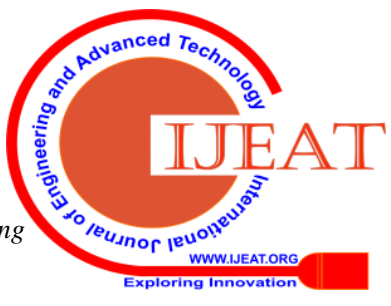

